\title{
Processed Panax ginseng, Sun Ginseng, Decreases Oxidative Damage Induced by tert-butyl Hydroperoxide via Regulation of Antioxidant Enzyme and Anti-apoptotic Molecules in HepG2 Cells
}

\author{
Hyejin Lee ${ }^{1}$, Jinhee Kim¹, Seo Young Lee², Jeong Hill Park², and Gwi Seo Hwang ${ }^{{ }^{*}}$ \\ ${ }^{1}$ Lab of Cell Differentiation Research, College of Oriental Medicine, Gachon University, Seongnam 461-701, Korea \\ ${ }^{2}$ College of Pharmacy, Seoul National University, Seoul 151-742, Korea
}

\begin{abstract}
Potential antioxidant effect of processed ginseng (sun ginseng, SG) on oxidative stress generated by tert-butyl hydroperoxide ( $t$-BHP) was investigated in HepG2 cells. 3-(4,5-Dimethylthiazol-2-yl)-2,5-diphenyltetrazolium bromide assay and lactate dehydrogenase (LDH) leakage test demonstrated that SG dose-dependently prevents a loss of cell viability against $t$-BHP-induced oxidative stress. Also, SG treatment dose-dependently relieved the increment of activities of hepatic enzymes, such as aspartate aminotrasferase and alanine aminotransferase, and lipid peroxidation mediated by $t$-BHP treatment in HepG2 cells. SG increased the gene expression of antioxidant enzymes such as superoxide dismutase, catalase, and glutathione peroxidase. However, high dose of SG treatment caused decrease in mRNA level of glutathione peroxidase as compared to low dosage of SG-treated cells. The gene expression of glutathione reductase was found to be slightly increased by SG treatment. In addition, SG extract attributed its hepaprotective effect by inducing the mRNA level of bcl-2 and bcl-xL but reducing that of bax. But, the gene expression of bad showed no significant change in SG-treated HepG2 cells. These findings suggest that SG has hepatoprotective effect by showing reduction of LDH release, activities of hepatic enzymes and lipid peroxidation and regulating the gene expression of antioxidant enzymes and apoptosis-related molecules against oxdative stress caused by $t$-BHP in HepG2 cells.
\end{abstract}

Keywords: Panax ginseng, Sun ginseng, Antioxidant activity, tert-butyl hydroperoxide, HepG2

\section{INTRODUCTION}

The balance between the production of reactive oxygen species (ROS) and the endogenous antioxidant system could prevent oxidative damage and induction of cell death. ROS accumulation caused by environmental factors, drugs, or pathological factors naturally occurred in cells. ROS, intermediates of oxidative metabolism, and other free radicals are intracellular factors related to pathogenesis of numerous diseases such as cancer, diabetes, neurodegenerative and cardiovascular diseases. It is reported that oxidative stress caused by ROS and

(c) This is an Open Access article distributed under the terms of the Creative Commons Attribution Non-Commercial License (http://creativecommons.org/licenses/by-nc/3.0/) which permits unrestricted non-commercial use, distribution, and reproduction in any medium, provided the original work is properly cited. reactive nitrogen species plays an important role in hepatic disease induction and progression $[1,2]$. Oxidative stress is responsible for the induction of the non-alcholic steatosis, alcoholic and viral hepatitis and hepatocellular carcinoma. Because the liver is central metabolic organ, metabolic events and blood flow from the gastrointestinal tract make the liver to be susceptible to absorbed drugs and environmental contaminants. These toxicants cause ROS generation and subsequently trigger apoptotic/ necrotic cell death leading to inflammatory and fibrotic

Received 21 Feb. 2012, Revised 13 Mar. 2012, Accepted 03 Apr. 2012

*Corresponding author

E-mail: seoul@gachon.ac.kr

Tel: +82-31-750-5421, Fax: +82-31-721-7029 
liver disorder and liver cancer. Natural antioxidants like vitamins, flavonoids and polyphenolic compounds have been proposed to possess anti-oxidative effect in liver and considered as therapeutic agents to counteract liver damage.

Panax ginseng Meyer is a widely used traditional herb in Asian countries having multi-functional activities such as anti-inflammatory, antioxidant, anti-tumor promoting and anti-aging potencies. Recently, the efficacy of ginseng is also regarded as a treatment for cardiovascular disease and diabetic complications [3,4]. Kim et al. [5] reported a new processed ginseng, named as sun ginseng (SG), which was prepared by steaming at higher temperature. As SG contains different types of ginsenosides including unique less-polar ginsenoside such as $\mathrm{Rg} 3$, $\mathrm{Rg} 5$ and $\mathrm{Rk} 1$, consequently possesses multi-therapeutic efficacy. In certain pathological conditions, SG represents more potent pharmacological effects than red ginseng (RG) or white ginseng (WG) [6]. There are a few reports about the beneficial effects of ginseng extract on hepatoprotection or hepatic antioxidative enzyme activities. American ginseng extracts which contain much watersoluble ginsenosides and materials have been reported to inhibit apoptosis through mediation of caspase- 9 and Bcl-2 activation [7]. Administration of the standardized $P$. ginseng extract G115 increased the antioxidant capacity with a reduction of the effects of exercise-induced oxidative stress in rats [8]. Also, it is reported that SG is hepatoprotective in terms of inhibition of cytochrome P450 and reduction of thiobarbituric acid-reactive substance in liver microsome prepared from carbon tetrachloridetreated rats [9]. Although various biological activities were reported for SG [10-13], the antioxidative and antiapoptotic effect of SG on HepG2 cell have yet to be evaluated.

HepG2 cells are well-established cell line to study the cytoprotective and anticarcinogenic effect of various natural compounds to liver cells $[14,15]$. As tert-butyl hydroperoxide ( $t$-BHP), an organic hydroperoxide, induces the release of arachidonic acid from cell membrane phospholipid and subsequent ROS-induced cell damage and the depletion of glutathione (GSH) and nicotinamide adenine dinucleotide phosphate (NADPH) leading to loss of cell viability, $t$-BHP is a hepatotoxic reagent which commonly used to induce the oxidative damages in HepG2 cells [16].

In this study, to investigate of the antioxidative and anti-apoptotic effect of SG on $t$-BHP-treated HepG2 cells, cell viability, hepatic enzymes activities and lipid peroxidation were evaluated. Also, the mRNA level of antioxidant enzymes and anti/pro-apoptotic molecules was measured to verify the hepatoprotective effect of SG.

\section{MATERIALS AND METHODS}

\section{Materials}

3-(4,5-Dimethylthiazol-2-yl)-2,5-diphenyltetrazolium bromide (MTT), $t$-BHP and 1,3,3-tetraethoxypropane (TEP) were purchased from Sigma Aldrich (St. Louis, MO, USA). Lactate dehydrogenase (LDH) assay kit was purchased from BCS Co. (Anyang, Korea). RNAiso Plus, a Primescript 1st strand cDNA synthesis kit, and a SYBR Premix Ex Taq real time polymerase chain reaction (PCR) kit were purchased from Takara Bio Inc. (Otsu, Japan). Extracts of SG, RG, and WG were provided by Ginseng Science Inc. (Seoul, Korea). Ginseng extracts were dissolved in dimethylsulfoxide (DMSO), passed through filter paper, and added to the cells at the indicated concentration.

\section{Cell culture}

HepG2 cell (Korean Cell Line Bank, Seoul, Korea) were grown at $37^{\circ} \mathrm{C}$ in DMEM medium (WelGENE, Daegu, Korea) supplemented with 10\% heat-inactivated fetal bovine serum (Lonza, Walkersville, MD, USA) and $1 \%$ antibiotics (Gibco BRL Life Technology, Grand Island, $\mathrm{NY}, \mathrm{USA}$ ) in a $5 \% \mathrm{CO}_{2}$-air atmosphere.

\section{3-(4,5-Dimethylthiazol-2-yl)-2,5-diphenyltetrazo- lium bromide assay}

To measure the extent of cell damage caused by $t$-BHP, cellular viability was determined by MTT assay. HepG2 cells at $5 \times 10^{4}$ cells were plated into 96 well-plates and incubated for $24 \mathrm{~h}$. Following incubation, cells were preincubated with extracts for $1 \mathrm{~h}$ and co-incubated with 2.5 $\mathrm{mM}$ of $t$-BHP to induce the cell death for $3 \mathrm{~h}$. And then medium was discarded and $0.5 \mathrm{mg} / \mathrm{mL}$ of MTT solution was added to each well. $3 \mathrm{~h}$ later, the formazan crystal formed in cells was dissolved by adding DMSO and the absorbance was measured at $570 \mathrm{~nm}$ by a microplate reader (Tecan, Grodig, Austria).

\section{Lactate dehydrogenase leakage assay}

To assess the effects of SG on $t$-BHP-induced oxidative stress in HepG2 cells, cell viability was measured by a LDH leakage assay using an assay kit according to the manufacturer's instructions. Cells were treated and incubated as described above. The culture media were used as an indicator of the plasma membrane integrity of cells. The enzyme activity was measured at $30^{\circ} \mathrm{C}$ and cell via- 
bility is represented as a value of LDH activity expressed in Wroblewski units.

\section{Aspartate aminotrasferase and alanine amino- transferase activity assay}

To evaluate the protective effects of SG on $t$-BHPinduced oxidative stress in HepG2 cells, aspartate aminotrasferase (AST) and alanine aminotransferase (ALT) activities were measured by using an assay kit according to the manufacturer's instructions. Cells were treated and incubated as described above. The culture media were used as an indicator of the normal function of liver. The enzyme activity was expressed in Karmen unit $/ \mathrm{mL}$ using calibration curve by plotting the corresponding absorbance of lithium pyruvate.

\section{Lipid peroxidation}

In order to estimate the lipid peroxidation, thiobarbituric acid reactive substance (TBARS) assay was performed. HepG2 cells at $5 \times 10^{4}$ cells were plated into 96 well-plates and incubated for $18 \mathrm{~h}$. Following incubation, medium was discarded and serum free fresh medium containing with 5,10 and $20 \mu \mathrm{g} / \mathrm{mL}$ of SG was treated for $1 \mathrm{~h}$ and then $2.5 \mathrm{mM}$ of $t$-BHP was added to induce oxidative stress. $3 \mathrm{~h}$ later, culture medium was precipitated with 50\% trichloroacetic acid and centrifuged at 1,500 rpm for $10 \mathrm{~min}$. After centrifuge, the supernatant was mixed with $0.67 \%$ thiobarbituric acid and boiled for 10 min. After cooling, the absorbance was measured at 535 $\mathrm{nm}$ by a microplate reader. The lipid peroxidation levels were showed by malondialdehyde (MDA) concentration made by the hydrolysis of TEP.

\section{RNA extraction and quantitative real time poly- merase chain reaction}

Total RNA was isolated by using RNAiso PLUS reagent (Takara Bio Inc.) according to the manufacturer's instructions. RNA concentrations and purity were determined using NanoDrop (NanoDrop Technologies Inc., Rockland, DE, USA). Total RNA was synthesized to first strand cDNA using a PrimeScript 1st strand cDNA synthsis kit. The cDNA was amplified to estimate the gene expression level by quantitative real time PCR. PCR reactions were performed using a SYBR premix Ex Taq kit and conducted using a Takara Dice Real Time System. All mRNA levels were normalized using the $\beta$-actin mRNA level as an internal control. The primers used in amplification are shown in Table 1.

\section{Statistical analysis}

Data were represented as the mean $\pm \mathrm{SD}$ and the significance of the results was assessed by Student's $t$-test (performed by the Sigmaplot computerized program). All experiments were conducted at least three times.

\section{RESULTS AND DISCUSSION}

\section{Sun ginseng protected HepG2 cells against tert- butyl hydroperoxide-induced oxidative damage}

To determine the effect of SG on $t$-BHP-induced oxidative stress, cell viability was measured by MTT assay. It has been reported that methanol extract of SG shows liver-protective effect on lipopolysaccharide-induced liver injury in rats by up-regulating hepatic inducible nitric oxide synthase and heme oxygenase 1 (HO-1) protein through NF- $\kappa \mathrm{B}$ deactivation [17]. Lee et al. [18] reported that the 20(S)-ginsenoside Rg3 and its metabolite, Rh2 isolated from $P$. ginseng have hepatoprotective effect by increasing cell viability in $t$-BHP treated HepG2 cells and $t$-BHP-induced liver injury of mice. In present studies, incubation with $2.5 \mathrm{mM}$ of $t$-BHP for $3 \mathrm{~h}$ induced about $50 \%$ cell death in HepG2 cells (Fig. 1A). Cell viability was recovered with increasing dosage of SG $(5,10$ and $20 \mu \mathrm{g} / \mathrm{mL}$ ) by $10,10.7,18.3$ and $25 \%$ respectively as

Table 1. Oligonucleotide primer sequences used in quantitative real time polymerase chain reaction

\begin{tabular}{llc}
\hline Gene name & \multicolumn{1}{c}{ Forward primer } & Reverse primer \\
\hline Superoxide dismutase & AGGCCGTGTGCGTGCTGAAG & CACCTTTGCCCAAGTCATCTGC \\
Catalase & CCTTTCTGTTGAAGATGCGGCG & GGCGGTGAGTGTCAGGATAG \\
Glutathione peroxidase & GTGTATGCCTTCTCGGCGCG & CGTTGCGACACACCGGAGAC \\
Glutathione reductase & CAGTGGGACTCACGGAAGAT & TTCACTGCAACAGCAAAACC \\
Bcl-2 & GATTGATGGGATCGTTGCCTTA & CCTTGGCATGAGATGCAGGA \\
Bcl-xL & CTTTGCCTAAGGCGGATTTGAA & AATAGGGATGGGCTCAACCAGTC \\
Bad & TGAGTGACGAGTTTGTGGACTCCT & CTGGAGCTTTGCCGCATCT \\
Bax & GCGAGTGTCTCAAGCGCATC & CCAGTTGAAGTTGCCGTCAGAA \\
-actin & TCACCCACACTGTGCCCAT & TCCTTAATGTCACGCACGATTT \\
\hline
\end{tabular}


A

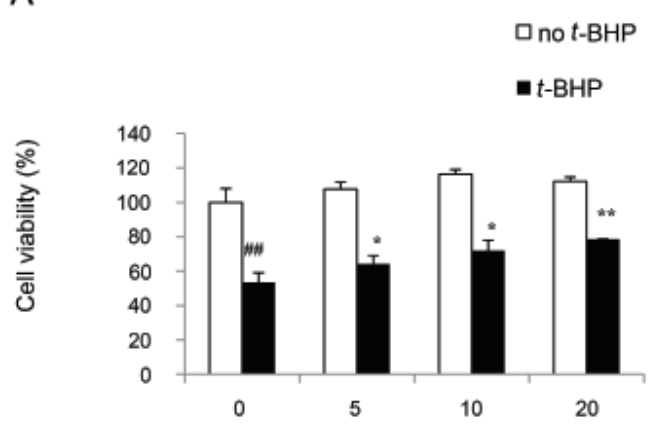

B

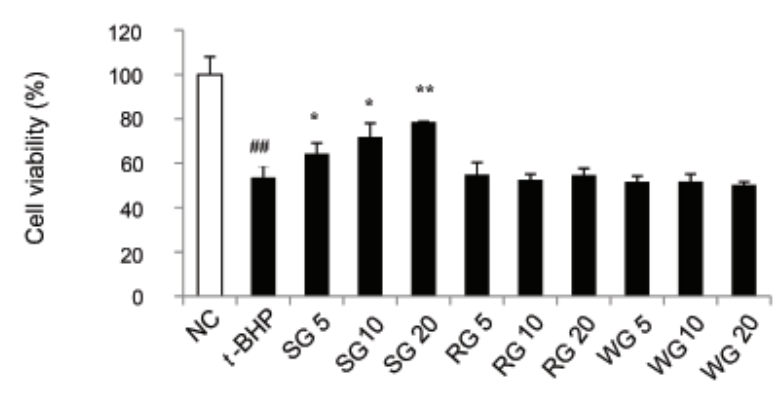

C

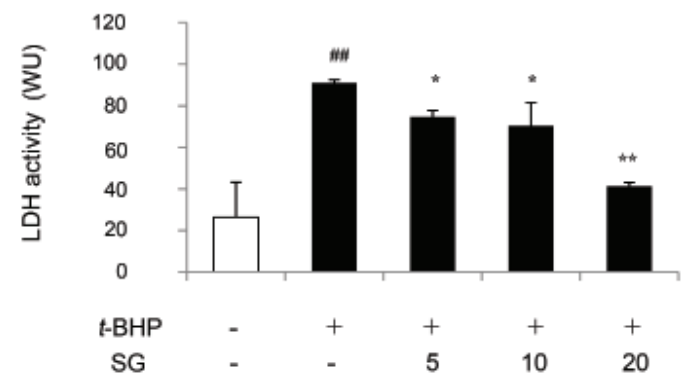

Fig. 1. Protective effects of sun ginseng (SG) against tert-butyl hydroperoxide ( $t$-BHP)-induced-oxidative stress in HepG2 cells. Cells were pretreated with the indicated concentration of $S G$, red ginseng (RG) or white ginseng (WG) $(5,10$, and $20 \mu \mathrm{g} / \mathrm{mL})$ and then exposed to $2.5 \mathrm{mM}$ of $t$-BHP for $3 \mathrm{~h}$. After incubation, 3-(4,5-dimethylthiazol2-yl)-2,5-diphenyltetrazolium bromide assay $(A, B)$ and lactate dehydrogenase (LDH) leakage assay $(C)$ were performed to determine the cell viability. Cell viability was expressed as a percentage of the negative control $(\mathrm{NC})$ in HepG2 cells $(\mathrm{A}, \mathrm{B})$ and a value of $\mathrm{LDH}$ activity expressed in Wroblewski units (WU) (C). Data represent the mean $\pm S D$ of triplicate experiments. Differences were considered significant at ${ }^{\#} p<0.01$ vs. $t$-BHP-untreated cells (NC) and ${ }^{*} p<0.05$ and ${ }^{* *} p<0.01$ vs. $t$-BHP-treated cells.

compared with $t$-BHP-treated control (Fig. 1A). When HepG2 cells were incubated in the absence of $t$-BHP, SG slightly increased cell viability, although not significantly. To compare the effect of SG with that of RG and WG, cells were pre-treated with SG, RG and WG extract for 1 $\mathrm{h}$, respectively. The same dosage of RG and WG did not show significant change in cell viability of HepG2 cells as compared with $t$-BHP-treated cells (Fig. 1B).

To confirm the potential hepatoprotective effect of SG against the $t$-BHP-induced toxicity, the cell viability was measured by LDH leakage assay (Fig. 1C). Consistent with MTT assay, increase in LDH release caused by $t$ BHP exposure was significantly reduced in HepG2 cells treated with SG up to $55.6 \%$ as compared to $t$-BHPtreated cells. These results suggest that SG can be a more potent antioxidative or hepatoprotective reagent than $\mathrm{RG}$ and WG.

\section{Sun ginseng reduced AST and ALT activities in tert- butyl hydroperoxide-damaged HepG2 cells}

In addition, the activity of two hepatic enzyme markers, serum AST and ALT, was determined using commercially available assay kits. Although the assessment of serum AST, ALT and alkaline phosphatase levels is commonly used as part of a diagnostic liver function test, AST and ALT have a broader clinical utility since it may also be elevated in diseases affecting other organs, such as the heart or muscles in myocardial infarction, in acute pancreatitis, acute hemolytic anemia, severe burns, acute renal disease, musculoskeletal diseases and trauma. As the elevation of AST and ALT activities caused by $t$-BHP treatment implies induction of hepatic damage in organs including liver, any compound decreasing these enzyme activities has hepatoprotective effect [18]. Jeong et al. [19] reported that administration of the standardized saponins of RG partially recovered the elevating serum AST and ALT activities induced by carbon tetrachloride in rat.

As shown in Fig. 2, $t$-BHP treatment dramatically increased AST and ALT activities in HepG2 cells. The SG pretreatment $(5,10$, and $20 \mu \mathrm{g} / \mathrm{mL})$ significantly inhibited AST activities (by 47, 63.6, and 77.2\%, respectively) in a dose-dependent manner as compared to $t$ BHP-treated HepG2 cells (Fig. 2A). The similar results were obtained in ALT activity assay (Fig. 2B). Increasing concentration of SG pretreatment down-regulated ALT activity up to $54.2 \%$, as compared to $t$-BHP-treated HepG2 cells. These data indicated that SG is a beneficial hepatoprotective reagent by ROS scavenging activity.

\section{Sun ginseng decreased tert-butyl hydroperoxide- induced lipid peroxidation in HepG2 cells}

ROS generation caused by $t$-BHP treatment leads to the oxidation of unsaturated fatty acid in lipids and subsequently produces intracellular ROS more [20]. The mobility rate of cell death and promotion of cancer will increase along with ROS-mediated damage to GSH, 


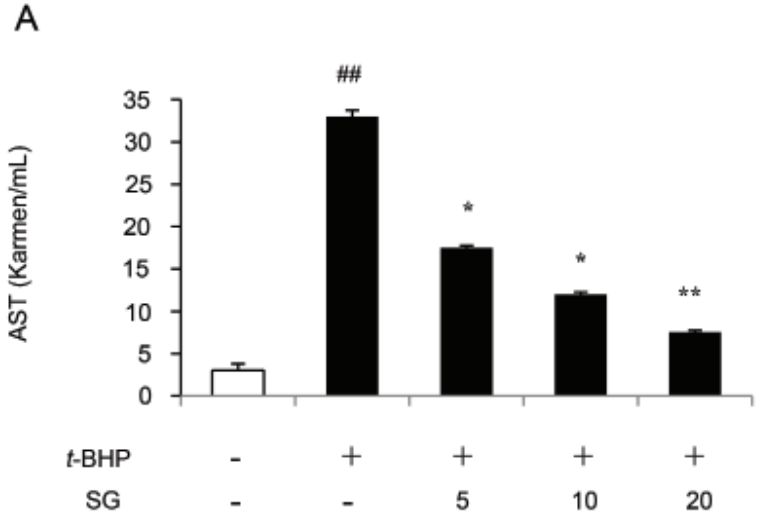

B

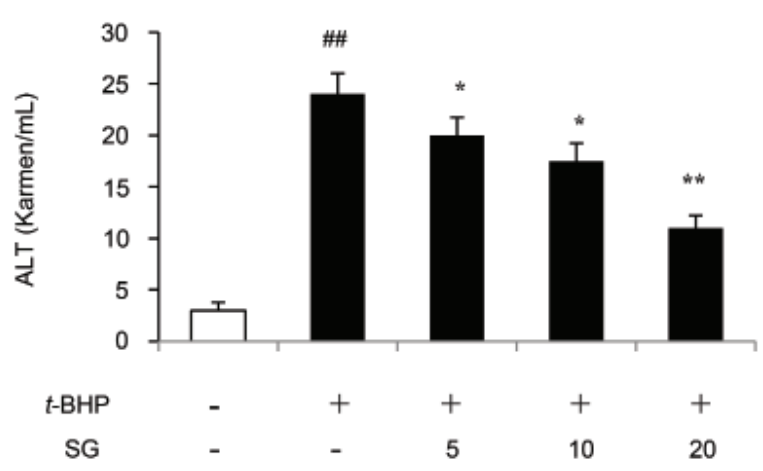

Fig. 2. Effect of sun ginseng (SG) on aspartate aminotrasferase (AST) and alanine aminotransferase (ALT) activities against tertbutyl hydroperoxide ( $t$-BHP)-induced-oxidative stress in HepG2 cells. Cells were pre-treated with the indicated concentration of SG $(5,10$, and $20 \mu \mathrm{g} / \mathrm{mL}$ ) and then exposed to $2.5 \mathrm{mM}$ of $t$-BHP for $3 \mathrm{~h}$ as mentioned. After incubation, AST (A) and ALT (B) activities were measured by using an assay kit according to the manufacturer's instructions. The enzyme activity was expressed in Karmen unit $/ \mathrm{mL}$. Data represent the mean $\pm S D$ of triplicate experiments. Differences were considered significant at ${ }^{\#} p<0.01$ vs. negative control and ${ }^{*} p<0.05$ and ${ }^{* *} p<0.01$ vs. $t$-BHP-treated cells.

proteins, DNA and lipids. To determine whether SG affects $t$-BHP-induced lipid peroxidation level, final lipid peroxidation product known as MDA concentration was measured by TBARS assay. Treatment with $2.5 \mathrm{mM}$ of $t$-BHP for $3 \mathrm{~h}$ showed $50 \%$ decrease of cell viability and highly increase of MDA levels indicating the treatment of $t$-BHP caused oxidative damage in HepG2 cell. However, pre-incubation with 5,10 and $20 \mu \mathrm{g} / \mathrm{mL}$ of SG significantly decreased the cellular MDA level by 3.14, 22.3 and $57.6 \%$ respectively, as compared to $t$-BHP-treated control as shown in Fig. 3. It reported that the prevention of lipid peroxidation by various phenolic compounds is significantly associated with protective potential against $t$-BHP-toxicity in HepG2 [15,21].

Although it is possible that other intracellular factors

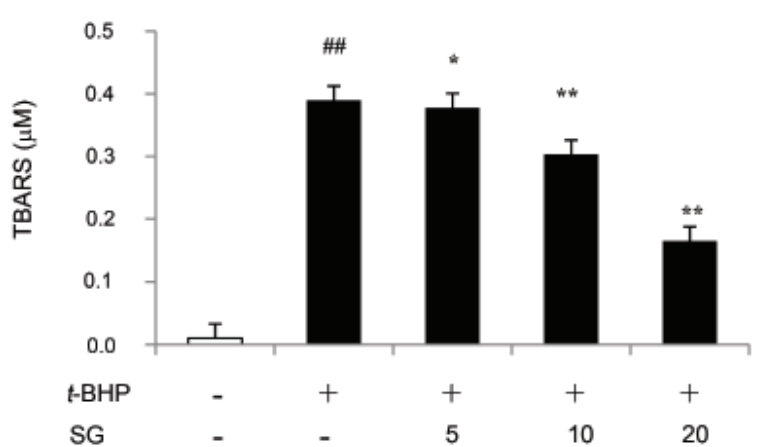

Fig. 3. Effect of sun ginseng (SG) on lipid peroxiation against tertbutyl hydroperoxide ( $t$-BHP)-induced-oxidative stress in HepG2 cells. Cells were pre-treated with the indicated concentration of SG (5, 10 , and $20 \mu \mathrm{g} / \mathrm{mL}$ ) and then exposed to $2.5 \mathrm{mM}$ of $t-B H P$ for $3 \mathrm{~h}$ as mentioned. After incubation, culture medium was precipitated with $50 \%$ trichloroacetic acid and thiobarbituric acid reactive substance (TBARS) assay was performed. The lipid peroxidation levels were showed by malondialdehyde concentration made by the hydrolysis of 1,3,3-tetraethoxypropane. Data represent the mean $\pm S D$ of triplicate experiments. Differences were considered significant at ${ }^{\#} p<0.01$ vs. negative control and ${ }^{*} p<0.05$ and ${ }^{* *} p<0.01$ vs. $t$-BHP-treated cells.

mediate protection pathway against $t$-BHP-induced oxidative damage in HepG2 cells [22,23], it is most likely that inhibition of lipid peroxidation plays role in antioxidative mechanism.

\section{Sun ginseng up-regulated gene expression of an- tioxidant enzymes}

Natural compounds having antioxidant activity contribute to induce the activity of intracellular antioxidant enzymes such as superoxide dismutase (SOD), catalase (CAT), glutathione peroxidase (GPx) and glutathione reductase (GR) $[24,25]$. The increase in these enzyme activities is considered to adaptive response to oxidative stress in cells. It was well known that incubation of HepG2 cells with $t$-BHP increased antioxidant enzyme activities [26].

In spite of many reports represented increment of antioxidant enzymes activities caused by $t$-BHP treatment, there are a few studies showing the change of gene expression of these enzymes. Our study demonstrated that SG dose-dependently increased the gene expression of SOD and CAT by real-time PCR analysis. SOD and CAT are important component of the cellular defense against oxidative stress by scavenging superoxide anion and hydrogen peroxide resulting in removal of ROS [26]. At a dose of $20 \mu \mathrm{g} / \mathrm{mL}$, SG up-regulated mRNA level of SOD and CAT by 12.4 and 9.4-fold as compared to untreated cells (negative control, NC) (Fig. 4). The effect of 5 and $10 \mu \mathrm{g} / \mathrm{mL}$ of SG treatment on the change in mRNA level of GPx correlated to its antioxidant effect showing incre- 


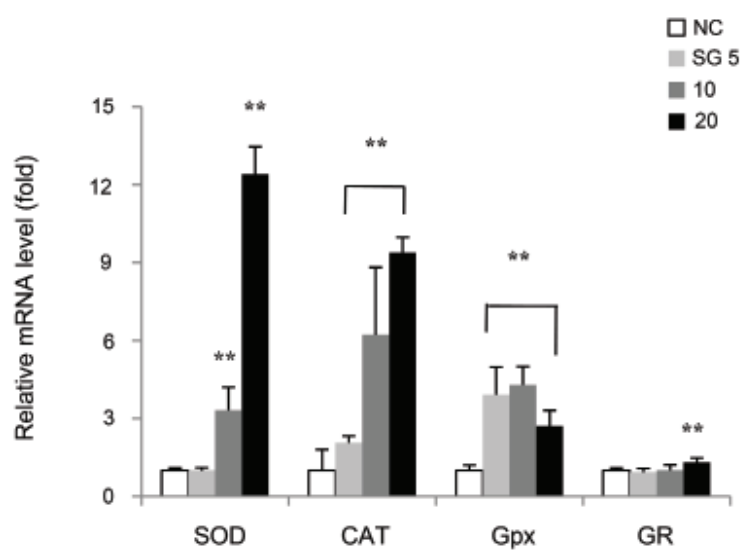

Fig. 4. Effect of sun ginseng (SG) on gene expression of antioxidant enzymes in HepG2 cells. To analyze the effects of SG on superoxide dismutase (SOD), catalase (CAT), glutathione peroxidase (GPx), and glutathione reductase (GR) gene expression by a real time polymerase chain reaction analysis, cells were incubated with the indicated concentration of SG $(5,10$, and $20 \mu \mathrm{g} / \mathrm{mL})$ for $24 \mathrm{~h}$. Data represent the mean $\pm S D$ of triplicate experiments. Differences were considered significant at ${ }^{*} p<0.05$ and ${ }^{* *} p<0.01$ vs. negative control (NC).

ment by 4-fold as compared to NC. Interestingly, it was found that $20 \mu \mathrm{g} / \mathrm{mL}$ of SG down-regulated mRNA level of GPx by 0.37 -fold as compared to $10 \mu \mathrm{g} / \mathrm{mL}$ of SG. CAT plays role in the detoxification of hydrogen peroxide by co-oxidizing glutathione which is catalyzed by GPx action. It is possible that the increase in mRNA level of CAT implies decrease in intracellular GSH content resulting in enhancement of oxidative stress and induction of hepatic damage. Because GSH acts as intracellular antioxidant, reduction of the gene expression of GPx may contribute to become cells less vulnerable to oxidative stress. Consistent with our data, Lee et al. [27] investigated that high dose of ginseng extract significantly reduced the GPx activity for maintaining intracellular GSH concentration in hydrogen peroxide-damaged $\mathrm{HepG} 2$ cells. On the other hand, mRNA level of GR slightly increased in this study. A glutathione disulfide can be reduced to the sulfhydryl form GSH by GR, using NADPH as an electron donor. The ratio of reduced GSH to oxidized glutathione within cells is regarded as an indicator of cellular toxicity. According to our results, an antioxidant effect of SG may be attributed to its potency of regulating the gene expression of antioxidant enzymes.

\section{Sun ginseng affected gene expression of mol- ecules related to apoptosis}

Treatment of $t$-BHP induces cell death resulting from oxidative DNA damage and/or disruption of mitochondrial membrane potential in different cell lines [28,29]. If

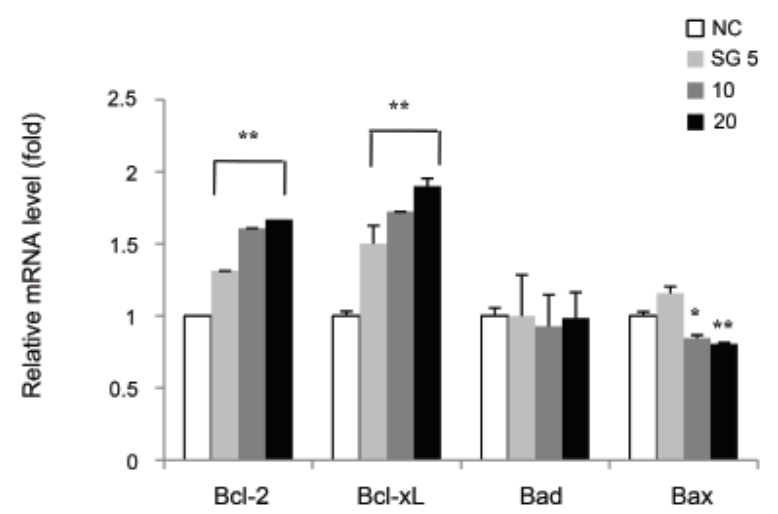

Fig. 5. Effect of sun ginseng (SG) on gene expression of anti- and pro-apoptotic molecules in HepG2 cells. To analyze the effects of SG on bcl-2, bcl-xL, bad, and bax gene expression by a real time polymerase chain reaction analysis, cells were incubated with the indicated concentration of SG $(5,10$, and $20 \mu \mathrm{g} / \mathrm{mL})$ for $24 \mathrm{~h}$. Data represent the mean $\pm S D$ of triplicate experiments. Differences were considered significant at ${ }^{*} p<0.05$ and ${ }^{* *} p<0.01$ vs. negative control (NC).

not removed by antioxidant enzymes such as SOD, CAT, GPx, GR and HO-1 and/or non-enzymatic substances such as GSH and vitamins, an excessive ROS generated by $t$-BHP is, therefore, able to induce the spontaneous and death receptor-mediated apoptosis.

As mentioned in other studies, protective effect of ginseng has been observed by controlling the mRNA and/ or protein level of molecules related to apoptosis. RG extract decreased the expression of pro-apoptotic protein, caspase- 3 and increased that of anti-apoptotic protein, bcl-2 to protect the human neuroblastoma, SK-N-SH cells against hydrogen peroxide-induced oxidative stress [30]. Also, water extract of American ginseng root has been investigated to prevent apoptosis by increasing the protein level of bcl-2 and decreasing that of caspase-9 in $\beta$ cells cultured with interleukin-1 $\beta$ [7].

In this study, the mRNA levels of pro- and antiapoptotic proteins belong to bcl-2 family were measured by real-time PCR analysis (Fig. 5). As the bcl-2 and bcl$\mathrm{xL}$ are anti-apoptotic molecules inhibiting cytochrome c release from mitochondria membrane, an increase in mRNA level of them serves as a marker of cells enduring apoptosis. In this study, SG dose-dependently increased bcl-2 mRNA up to 1.7-fold in HepG2 cells as compared to NC. We further tested whether the mRNA level of bcl$\mathrm{xL}$, another bcl- 2 family member, was also changed by SG treatment. SG $(5,10$, and $20 \mu \mathrm{g} / \mathrm{mL})$ significantly increased bcl-xL expression by 1.5-, 1.7- and 1.9-fold, respectively, as compared to NC. In addition, the mRNA level of pro-apoptotic proteins, bad and bax, was evaluated in the presence of SG. 5,10 , and $20 \mu \mathrm{g} / \mathrm{mL}$ of SG 
treatment had no significant change in mRNA level of bad as compared to NC. But, the slight decrease in mRNA level of bax was determined by 10 and $20 \mu \mathrm{g} /$ $\mathrm{mL}$ of SG treatment. Our results suggested that indicated concentration of SG was endowed with cytoprotective effect by inducing gene expression of bcl-2 and -xL and reducing that of bax in HepG2 cells.

In summary, our data demonstrated that SG has hepatoprotective effect by showing reduction of $\mathrm{LDH}$ release, activities of hepatic enzymes and lipid peroxidation against oxidative stress caused by $t$-BHP in HepG2 cells. The gene expression of antioxidant enzymes appeared to be increased with SG treatment, even when a higher dose of SG decreased mRNA level of GPx. The reduction in mRNA level of GPx is thought to be responsible for maintaining cellular GSH content. It was presumed that SG regulates the mRNA level of intracellular antioxidant enzymes to defense oxidative stress in HepG2 cells. In addition, the SG treatment increased the mRNA level of anti-apoptotic molecules, bcl-2 and bcl-xL. It correlated inversely with decrease in mRNA level of apoptotic molecule, bax, without affecting bad expression.

\section{ACKNOWLEDGEMENTS}

The authors wish to acknowledge the financial support of Gachon University Research Fund (GCU 2012-R004).

\section{REFERENCES}

1. Vitaglione P, Morisco F, Caporaso N, Fogliano V. Dietary antioxidant compounds and liver health. Crit Rev Food Sci Nutr 2004;44:575-586.

2. Adachi M, Ishii H. Role of mitochondria in alcoholic liver injury. Free Radic Biol Med 2002;32:487-491.

3. Kim HJ, Chae IG, Lee SG, Jeong HJ, Lee EJ, Lee IS. Effects of Fermented Red Ginseng Extracts on Hyperglycemia in Streptozotocin-induced Diabetic Rats. J Ginseng Res 2010;34:104-112.

4. Hur MH, Lee MS, Yang HJ, Kim C, Bae IL, Ernst E. Ginseng for Reducing the Blood Pressure in Patients with Hypertension: A Systematic Review and Meta-Analysis. J Ginseng Res 2010;34:342-347.

5. Kim WY, Kim JM, Han SB, Lee SK, Kim ND, Park MK, Kim CK, Park JH. Steaming of ginseng at high temperature enhances biological activity. J Nat Prod 2000;63:1702-1704.

6. Song KC, Chang TS, Lee H, Kim J, Park JH, Hwang GS. Processed Panax ginseng, sun ginseng increases type I collagen by regulating MMP-1 and TIMP-1 expression in human dermal fibroblasts. J Ginseng Res 2012;36:61-67.

7. Luo JZ, Luo L. American ginseng stimulates insulin production and prevents apoptosis through regulation of uncoupling protein-2 in cultured beta cells. Evid Based Complement Alternat Med 2006;3:365-372.

8. Voces J, Alvarez AI, Vila L, Ferrando A, Cabral de Oliveira C, Prieto JG. Effects of administration of the standardized Panax ginseng extract G115 on hepatic antioxidant function after exhaustive exercise. Comp Biochem Physiol C Pharmacol Toxicol Endocrinol 1999;123:175-184.

9. Kim HJ, Chun YJ, Park JD, Kim SI, Roh JK, Jeong TC. Protection of rat liver microsomes against carbon tetrachloride-induced lipid peroxidation by red ginseng saponin through cytochrome P450 inhibition. Planta Med 1997;63:415-418.

10. Park IH, Piao LZ, Kwon SW, Lee YJ, Cho SY, Park MK, Park JH. Cytotoxic dammarane glycosides from processed ginseng. Chem Pharm Bull (Tokyo) 2002;50:538540.

11. Baek SH, Piao XL, Lee UJ, Kim HY, Park JH. Reduction of cisplatin-induced nephrotoxicity by ginsenosides isolated from processed ginseng in cultured renal tubular cells. Biol Pharm Bull 2006;29:2051-2055.

12. Lee JG, Lee YY, Kim SY, Pyo JS, Yun-Choi HS, Park JH. Platelet antiaggregating activity of ginsenosides isolated from processed ginseng. Pharmazie 2009;64:602-604.

13. Kang KS, Yamabe N, Kim HY, Park JH, Yokozawa T. Effects of heat-processed ginseng and its active component ginsenoside $20(\mathrm{~S})-\mathrm{Rg} 3$ on the progression of renal damage and dysfunction in type 2 diabetic Otsuka Long-Evans Tokushima Fatty rats. Biol Pharm Bull 2010;33:10771081.

14. Sarkar MK, Sil PC. Prevention of tertiary butyl hydroperoxide induced oxidative impairment and cell death by a novel antioxidant protein molecule isolated from the herb, Phyllanthus niruri. Toxicol In Vitro 2010;24:1711-1719.

15. Lima CF, Fernandes-Ferreira M, Pereira-Wilson C. Phenolic compounds protect HepG2 cells from oxidative damage: relevance of glutathione levels. Life Sci 2006;79:2056-2068.

16. Lima CF, Valentao PC, Andrade PB, Seabra RM, Fernandes-Ferreira $\mathrm{M}$, Pereira-Wilson $\mathrm{C}$. Water and methanolic extracts of Salvia officinalis protect HepG2 cells from $t$-BHP induced oxidative damage. Chem Biol Interact 2007;167:107-115.

17. Kang KS, Yamabe N, Kim HY, Yokozawa T. Effect of sun ginseng methanol extract on lipopolysaccharide-induced liver injury in rats. Phytomedicine 2007;14:840-845.

18. Lee HU, Bae EA, Han MJ, Kim DH. Hepatoprotective effect of 20(S)-ginsenosides Rg3 and its metabolite 20(S)- 
ginsenoside $\mathrm{Rh} 2$ on tert-butyl hydroperoxide-induced liver injury. Biol Pharm Bull 2005;28:1992-1994.

19. Jeong TC, Kim HJ, Park JI, Ha CS, Park JD, Kim SI, Roh JK. Protective effects of red ginseng saponins against carbon tetrachloride-induced hepatotoxicity in Sprague Dawley rats. Planta Med 1997;63:136-140.

20. Fernandes ER, Carvalho FD, Remiao FG, Bastos ML, Pinto MM, Gottlieb OR. Hepatoprotective activity of xanthones and xanthonolignoids against tert-butylhydroperoxide-induced toxicity in isolated rat hepatocytes: comparison with silybin. Pharm Res 1995;12:1756-1760.

21. Vidyashankar S, Mitra SK, Nandakumar KS. Liv.52 protects HepG2 cells from oxidative damage induced by tertbutyl hydroperoxide. Mol Cell Biochem 2010;333:41-48.

22. Nicotera P, McConkey D, Svensson SA, Bellomo G, Orrenius S. Correlation between cytosolic $\mathrm{Ca}^{2+}$ concentration and cytotoxicity in hepatocytes exposed to oxidative stress. Toxicology 1988;52:55-63.

23. Martin C, Martinez R, Navarro R, Ruiz-Sanz JI, Lacort M, Ruiz-Larrea MB. tert-butyl hydroperoxide-induced lipid signaling in hepatocytes: involvement of glutathione and free radicals. Biochem Pharmacol 2001;62:705-712.

24. Lima CF, Andrade PB, Seabra RM, Fernandes-Ferreira M, Pereira-Wilson C. The drinking of a Salvia officinalis infusion improves liver antioxidant status in mice and rats.
J Ethnopharmacol 2005;97:383-389.

25. Noh JR, Gang GT, Kim YH, Yang KJ, Lee CH, Na OS, Kim GJ, Oh WK, Lee YD. Desalinated underground seawater of Jeju Island (Korea) improves lipid metabolism in mice fed diets containing high fat and increases antioxidant potential in $t$-BHP treated HepG2 cells. Nutr Res Pract 2010;4:3-10.

26. Sies H. Strategies of antioxidant defense. Eur J Biochem 1993;215:213-219.

27. Lee M, Sorn S, Baek S, Jang S, Kim S. Antioxidant and apoptotic effects of Korean white ginseng extracted with the same ratio of protopanaxadiol and protopanaxatriol saponins in human hepatoma HepG2 cells. Ann N Y Acad Sci 2009;1171:217-227.

28. Hix S, Kadiiska MB, Mason RP, Augusto O. In vivo metabolism of tert-butyl hydroperoxide to methyl radicals. EPR spin-trapping and DNA methylation studies. Chem Res Toxicol 2000;13:1056-1064.

29. Byrne AM, Lemasters JJ, Nieminen AL. Contribution of increased mitochondrial free $\mathrm{Ca} 2+$ to the mitochondrial permeability transition induced by tert-butylhydroperoxide in rat hepatocytes. Hepatology 1999;29:1523-1531.

30. Kim EH, Lee MJ, Kim IH, Pyo S, Choi KT, Rhee DK. Anti-apoptotic effects of red ginseng on oxidative stress induced by hydrogen peroxide in SK-N-SH cells. J Ginseng Res 2010;34:138-144. 